\title{
How Surfaces Affect Hybridization Kinetics
}

\section{SUPPORTING INFORMATION}

Eshan Treasurer, Rastislav Levicky*

Department of Chemical and Biomolecular Engineering, New York University Tandon School of

Engineering, Brooklyn, NY 11201, USA

*To whom correspondence should be addressed. Tel: 646-997-3682. E-mail:

RL1306@nyu.edu. 
TABLE S1. Kinetic data for solution hybridization, including experimental $k_{\mathrm{F}, \mathrm{B}}$ values and corresponding Wetmur-Davidson (WD) and Weighted Neighbor Voting (WNV) predictions.

\begin{tabular}{|c|c|c|c|c|c|c|c|c|c|}
\hline ref. & strand $1^{1}$ & $\begin{array}{c}\text { length } \\
\mathrm{nt} \\
\end{array}$ & $\begin{array}{l}T \\
{ }^{\circ} \mathrm{C} \\
\end{array}$ & $\begin{array}{c}{[\mathrm{Na}+]} \\
M \\
\end{array}$ & $\begin{array}{c}k_{\mathrm{F}, \mathrm{B}} \exp . \\
\mathrm{M}^{-1} \mathrm{~s}^{-1}\end{array}$ & $\begin{array}{r}k_{\mathrm{F}, \mathrm{B}} \text { WD } \\
\mathrm{M}^{-1} \mathrm{~s}^{-1} \\
\end{array}$ & $\begin{array}{c}k_{\mathrm{F}, \mathrm{B}} \mathrm{WNV} \\
\mathrm{M}^{-1} \mathrm{~s}^{-1}\end{array}$ & $\begin{array}{l}\Delta G_{\text {fold }}^{o}{ }^{2} \\
\text { strand } 1 \\
\mathrm{kcal} / \mathrm{mol}\end{array}$ & $\begin{array}{c}\Delta G_{\text {fold }}^{o} \\
{\text { strand } 2^{1}}^{1} \\
\mathrm{kcal} / \mathrm{mol}\end{array}$ \\
\hline 60 & GCGCGC & 6 & 25 & 1.0 & $\begin{array}{c}1.20 \mathrm{E}+ \\
07\end{array}$ & $\begin{array}{c}2.19 \mathrm{E}+ \\
06 \\
\end{array}$ & $\begin{array}{c}6.11 \mathrm{E}+0 \\
6\end{array}$ & $\mathrm{NSP}^{3}$ & $\mathrm{NSP}^{3}$ \\
\hline 61 & CAAAAAG & 7 & 20 & 1.0 & $\begin{array}{c}9.00 \mathrm{E}+ \\
06 \\
\end{array}$ & $\begin{array}{c}2.41 \mathrm{E}+ \\
06\end{array}$ & $\begin{array}{c}4.97 \mathrm{E}+0 \\
6 \\
\end{array}$ & $\mathrm{NSP}^{3}$ & $\mathrm{NSP}^{3}$ \\
\hline 62 & GCATGC & 6 & 31.1 & 1.0 & $\begin{array}{c}9.90 \mathrm{E}+ \\
06 \\
\end{array}$ & $\begin{array}{c}2.55 \mathrm{E}+ \\
06\end{array}$ & $\begin{array}{c}4.88 \mathrm{E}+0 \\
6 \\
\end{array}$ & $\mathrm{NSP}^{3}$ & $\mathrm{NSP}^{3}$ \\
\hline 44 & $\begin{array}{l}\text { GTTGTCAAGATG } \\
\text { CTACCGTTCAGA } \\
\text { G }\end{array}$ & 25 & 20 & 0.5 & $\begin{array}{c}1.20 \mathrm{E}+ \\
06\end{array}$ & $\begin{array}{c}1.02 \mathrm{E}+ \\
07\end{array}$ & $\begin{array}{c}2.61 \mathrm{E}+0 \\
6\end{array}$ & 0.1 & -0.2 \\
\hline 44 & $\begin{array}{l}\text { GTTGTCAAGATG } \\
\text { CTACCGTTCAGA } \\
\text { G }\end{array}$ & 25 & 20 & 1.0 & $\begin{array}{l}1.56 \mathrm{E}^{+} \\
06\end{array}$ & $\begin{array}{c}1.63 \mathrm{E}^{+} \\
07\end{array}$ & $\begin{array}{c}1.58 \mathrm{E}+0 \\
6\end{array}$ & 0 & -0.3 \\
\hline 44 & $\begin{array}{c}\text { AGATCAGTGCGT } \\
\text { CTGTACTAGCAG } \\
\mathrm{T}\end{array}$ & 25 & 20 & 0.5 & $\begin{array}{l}7.20 \mathrm{E}+ \\
05\end{array}$ & $\begin{array}{l}1.02 \mathrm{E}^{+} \\
07\end{array}$ & $\begin{array}{c}3.82 \mathrm{E}+0 \\
5\end{array}$ & -2.9 & -3.6 \\
\hline 44 & $\begin{array}{c}\text { AGATCAGTGCGT } \\
\text { CTGTACTAGCAG } \\
\mathrm{T} \\
\end{array}$ & 25 & 20 & 1.0 & $\begin{array}{c}8.60 \mathrm{E}+ \\
05\end{array}$ & $\begin{array}{c}1.63 \mathrm{E}^{+} \\
07\end{array}$ & $\begin{array}{c}3.61 \mathrm{E}+0 \\
5\end{array}$ & -3.2 & -3.9 \\
\hline 44 & $\begin{array}{c}\text { AGATCAGTGCGT } \\
\text { CTGTACTAGCAC } \\
\text { A }\end{array}$ & 25 & 20 & 0.5 & $\begin{array}{l}2.00 \mathrm{E}+ \\
05\end{array}$ & $\begin{array}{c}1.02 \mathrm{E}+ \\
07\end{array}$ & $\begin{array}{c}2.43 \mathrm{E}+0 \\
5\end{array}$ & -5 & -4.3 \\
\hline 44 & $\begin{array}{c}\text { AGATCAGTGCGT } \\
\text { CTGTACTAGCAC } \\
\text { A }\end{array}$ & 25 & 20 & 1.0 & $\begin{array}{l}4.30 \mathrm{E}+ \\
05\end{array}$ & $\begin{array}{c}1.63 \mathrm{E}+ \\
07\end{array}$ & $\begin{array}{l}4.00 \mathrm{E}+0 \\
5\end{array}$ & -5.3 & -4.6 \\
\hline 63 & $\begin{array}{l}\text { GCCCACACTCTT } \\
\text { ACTTATCGACT }\end{array}$ & 23 & 25 & 0.195 & $\begin{array}{c}3.50 \mathrm{E}+ \\
06\end{array}$ & $\begin{array}{c}1.93 \mathrm{E}+ \\
06\end{array}$ & $\begin{array}{c}1.03 \mathrm{E}+0 \\
7 \\
\end{array}$ & 1.9 & 1.5 \\
\hline 63 & $\begin{array}{l}\text { AGAGGCTTATAA } \\
\text { CTGTGTCGGGT }\end{array}$ & 23 & 25 & 0.195 & $\begin{array}{c}2.10 \mathrm{E}+ \\
06\end{array}$ & $\begin{array}{c}1.93 \mathrm{E}+ \\
06\end{array}$ & $\begin{array}{c}1.06 \mathrm{E}+0 \\
7\end{array}$ & 0.3 & 1.1 \\
\hline 63 & $\begin{array}{l}\text { TGTTCTAAGATT } \\
\text { ATCCTCCCGCC }\end{array}$ & 23 & 25 & 0.195 & $\begin{array}{c}2.21 \mathrm{E}+ \\
06\end{array}$ & $\begin{array}{c}1.93 \mathrm{E}+ \\
06\end{array}$ & $\begin{array}{c}3.82 \mathrm{E}+0 \\
6\end{array}$ & 1.1 & 0.6 \\
\hline 63 & $\begin{array}{c}\text { GGCGGCTATAAC } \\
\text { AATTTCATCCA }\end{array}$ & 23 & 25 & 0.195 & $\begin{array}{c}2.80 \mathrm{E}+ \\
06 \\
\end{array}$ & $\begin{array}{c}1.93 \mathrm{E}+ \\
06 \\
\end{array}$ & $\begin{array}{c}3.85 \mathrm{E}+0 \\
6 \\
\end{array}$ & 0.5 & 0.9 \\
\hline 63 & $\begin{array}{c}\text { TAGCCCAGTGAT } \\
\text { TTATGACATGC }\end{array}$ & 23 & 25 & 0.195 & $\begin{array}{c}4.30 \mathrm{E}+ \\
05 \\
\end{array}$ & $\begin{array}{c}1.93 \mathrm{E}+ \\
06 \\
\end{array}$ & $\begin{array}{c}4.00 \mathrm{E}+0 \\
6 \\
\end{array}$ & 0.5 & 0.8 \\
\hline 63 & $\begin{array}{l}\text { GCATCTACACTC } \\
\text { AATACCCAGCC }\end{array}$ & 23 & 25 & 0.195 & $\begin{array}{c}4.30 \mathrm{E}+ \\
06\end{array}$ & $\begin{array}{c}1.93 \mathrm{E}+ \\
06 \\
\end{array}$ & $\begin{array}{c}3.92 \mathrm{E}+0 \\
6 \\
\end{array}$ & 1.1 & 0.8 \\
\hline 63 & $\begin{array}{l}\text { GCCCGTACTGTT } \\
\text { GAGATTATGGT }\end{array}$ & 23 & 25 & 0.195 & $\begin{array}{c}9.20 \mathrm{E}+ \\
05\end{array}$ & $\begin{array}{c}1.93 \mathrm{E}+ \\
06\end{array}$ & $\begin{array}{c}4.75 \mathrm{E}+0 \\
6\end{array}$ & -0.4 & 0.3 \\
\hline 63 & $\begin{array}{l}\text { GCACCTCCAAAT } \\
\text { AAAAACTCCGC }\end{array}$ & 23 & 25 & 0.195 & $\begin{array}{c}4.80 \mathrm{E}+ \\
06 \\
\end{array}$ & $\begin{array}{c}1.93 \mathrm{E}+ \\
06 \\
\end{array}$ & $\begin{array}{c}8.87 \mathrm{E}+0 \\
6 \\
\end{array}$ & 1.6 & 0.1 \\
\hline 63 & $\begin{array}{c}\text { AGATCAGAGAT } \\
\text { AGTTACGCCGCA }\end{array}$ & 23 & 25 & 0.195 & $\begin{array}{c}1.25 \mathrm{E}+ \\
06 \\
\end{array}$ & $\begin{array}{c}1.93 \mathrm{E}+ \\
06 \\
\end{array}$ & $\begin{array}{c}5.95 \mathrm{E}+0 \\
6 \\
\end{array}$ & 0.2 & 0.3 \\
\hline 63 & $\begin{array}{l}\text { TATGTTCCTTAC } \\
\text { CCCGTTTACCA }\end{array}$ & 23 & 25 & 0.195 & $\begin{array}{c}7.30 \mathrm{E}+ \\
05\end{array}$ & $\begin{array}{c}1.93 \mathrm{E}+ \\
06\end{array}$ & $\begin{array}{c}3.83 \mathrm{E}+0 \\
6 \\
\end{array}$ & 0.3 & -0.6 \\
\hline 63 & TAGCCAACTCTA & 23 & 25 & 0.195 & $1.02 \mathrm{E}+$ & $1.93 \mathrm{E}+$ & $3.79 \mathrm{E}+0$ & 0 & 0.2 \\
\hline
\end{tabular}




\begin{tabular}{|c|c|c|c|c|c|c|c|c|c|}
\hline & AATAACGGACG & & & & 06 & 06 & 6 & & \\
\hline 63 & $\begin{array}{l}\text { GAAGGAATGTTA } \\
\text { AAATCGTCGCG }\end{array}$ & 23 & 25 & 0.195 & $\begin{array}{c}7.70 \mathrm{E}+ \\
05\end{array}$ & $\begin{array}{c}1.93 \mathrm{E}+ \\
06\end{array}$ & $\begin{array}{c}1.00 \mathrm{E}+0 \\
7\end{array}$ & 0.4 & 0.2 \\
\hline 63 & $\begin{array}{l}\text { TTTGTTTTCCTTA } \\
\text { TGAGCCAGCC }\end{array}$ & 23 & 25 & 0.195 & $\begin{array}{c}1.43 \mathrm{E}+ \\
06\end{array}$ & $\begin{array}{c}1.93 \mathrm{E}+ \\
06\end{array}$ & $\begin{array}{c}3.83 \mathrm{E}+0 \\
6\end{array}$ & 0.6 & 0.1 \\
\hline 63 & $\begin{array}{l}\text { GCCCCGATATCT } \\
\text { ATTTTAGGACG }\end{array}$ & 23 & 25 & 0.195 & $\begin{array}{c}1.35 \mathrm{E}+ \\
06 \\
\end{array}$ & $\begin{array}{c}1.93 \mathrm{E}^{+} \\
06 \\
\end{array}$ & $\begin{array}{c}4.96 \mathrm{E}+0 \\
6 \\
\end{array}$ & -0.1 & -0.4 \\
\hline 63 & $\begin{array}{l}\text { CGCAGGAGAGTT } \\
\text { AAACGAAAGCA }\end{array}$ & 23 & 25 & 0.195 & $\begin{array}{c}1.04 \mathrm{E}+ \\
05\end{array}$ & $\begin{array}{c}1.93 \mathrm{E}+ \\
06\end{array}$ & $\begin{array}{c}3.47 \mathrm{E}+0 \\
6\end{array}$ & 0 & 0.4 \\
\hline 63 & $\begin{array}{l}\text { GGCTCTATACGA } \\
\text { TTAAACTCCCC }\end{array}$ & 23 & 25 & 0.195 & $\begin{array}{c}1.55 \mathrm{E}+ \\
06\end{array}$ & $\begin{array}{c}1.93 \mathrm{E}+ \\
06\end{array}$ & $\begin{array}{c}3.84 \mathrm{E}+0 \\
6\end{array}$ & 1 & 0.2 \\
\hline 63 & $\begin{array}{c}\text { CATCTGAACGAG } \\
\text { TAAGGACCCCA }\end{array}$ & 23 & 25 & 0.195 & $\begin{array}{c}1.21 \mathrm{E}+ \\
06\end{array}$ & $\begin{array}{c}1.93 \mathrm{E}+ \\
06\end{array}$ & $\begin{array}{c}3.37 \mathrm{E}+0 \\
6\end{array}$ & -0.1 & -0.3 \\
\hline 63 & $\begin{array}{l}\text { CGTCTATTGCTT } \\
\text { GTCACTTCCCC }\end{array}$ & 23 & 25 & 0.195 & $\begin{array}{c}5.23 \mathrm{E}+ \\
05\end{array}$ & $\begin{array}{c}1.93 \mathrm{E}+ \\
06\end{array}$ & $\begin{array}{c}3.83 \mathrm{E}+0 \\
6\end{array}$ & 0.8 & 0.6 \\
\hline 63 & $\begin{array}{l}\text { AGTCCTTGGTTA } \\
\text { TCATTCCCTCT }\end{array}$ & 23 & 25 & 0.195 & $\begin{array}{c}1.05 \mathrm{E}+ \\
06\end{array}$ & $\begin{array}{c}1.93 \mathrm{E}+ \\
06\end{array}$ & $\begin{array}{c}6.43 \mathrm{E}+0 \\
6\end{array}$ & 0.1 & -0.5 \\
\hline 63 & $\begin{array}{c}\text { GGTCTCAGCTAA } \\
\text { TTTCACACAGA }\end{array}$ & 23 & 25 & 0.195 & $\begin{array}{c}6.30 \mathrm{E}+ \\
05\end{array}$ & $\begin{array}{c}1.93 \mathrm{E}+ \\
06\end{array}$ & $\begin{array}{c}3.83 \mathrm{E}+0 \\
6\end{array}$ & 0.6 & 0.6 \\
\hline 63 & $\begin{array}{l}\text { TGGGGGGCATA } \\
\text { AAACGATACTAG }\end{array}$ & 23 & 25 & 0.195 & $\begin{array}{c}4.70 \mathrm{E}+ \\
05\end{array}$ & $\begin{array}{l}1.93 \mathrm{E}+ \\
06\end{array}$ & $\begin{array}{c}9.84 \mathrm{E}+0 \\
6\end{array}$ & 0.4 & 0.6 \\
\hline 63 & $\begin{array}{l}\text { TGCTCACTTACA } \\
\text { TTACGTCCATG }\end{array}$ & 23 & 25 & 0.195 & $\begin{array}{c}7.00 \mathrm{E}+ \\
05\end{array}$ & $\begin{array}{c}1.93 \mathrm{E}+ \\
06\end{array}$ & $\begin{array}{c}6.15 E+0 \\
6\end{array}$ & 0.1 & -0.2 \\
\hline 63 & $\begin{array}{l}\text { ACCCTTTATCCT } \\
\text { GTAACTTCCGC }\end{array}$ & 23 & 25 & 0.195 & $\begin{array}{c}2.60 \mathrm{E}+ \\
06\end{array}$ & $\begin{array}{l}1.93 \mathrm{E}+ \\
06\end{array}$ & $\begin{array}{c}5.34 \mathrm{E}+0 \\
6\end{array}$ & 0.9 & -0.1 \\
\hline 63 & $\begin{array}{l}\text { GCCTAGTGAAAC } \\
\text { CGTAAGTGCAT }\end{array}$ & 23 & 25 & 0.195 & $\begin{array}{c}1.73 \mathrm{E}^{+} \\
05\end{array}$ & $\begin{array}{c}1.93 \mathrm{E}+ \\
06\end{array}$ & $\begin{array}{l}4.65 \mathrm{E}+0 \\
6\end{array}$ & 0 & 0.3 \\
\hline 63 & $\begin{array}{l}\text { TCAGCACTCTAC } \\
\text { TTGACGGACTT }\end{array}$ & 23 & 25 & 0.195 & $\begin{array}{c}8.70 \mathrm{E}^{+} \\
04\end{array}$ & $\begin{array}{c}1.93 \mathrm{E}+ \\
06\end{array}$ & $\begin{array}{c}4.75 \mathrm{E}+0 \\
6\end{array}$ & 0.5 & -0.1 \\
\hline 63 & $\begin{array}{c}\text { AGGTTAGGATTT } \\
\text { GTCGGGAGATG }\end{array}$ & 23 & 25 & 0.195 & $\begin{array}{c}3.10 \mathrm{E}+ \\
06\end{array}$ & $\begin{array}{c}1.93 \mathrm{E}+ \\
06\end{array}$ & $\begin{array}{c}1.15 \mathrm{E}+0 \\
7 \\
\end{array}$ & -0.3 & 0.6 \\
\hline 63 & $\begin{array}{l}\text { GTCCCGGAAAAT } \\
\text { ACTATGAGACC }\end{array}$ & 23 & 25 & 0.195 & $\begin{array}{c}3.70 \mathrm{E}+ \\
04\end{array}$ & $\begin{array}{c}1.93 \mathrm{E}+ \\
06\end{array}$ & $\begin{array}{c}1.79 \mathrm{E}+0 \\
6\end{array}$ & 0 & -0.2 \\
\hline 63 & $\begin{array}{l}\text { GGCGCTTAAATC } \\
\text { ATCTTTCATCG }\end{array}$ & 23 & 25 & 0.195 & $\begin{array}{c}5.90 \mathrm{E}+ \\
05 \\
\end{array}$ & $\begin{array}{c}1.93 \mathrm{E}+ \\
06 \\
\end{array}$ & $\begin{array}{c}1.70 \mathrm{E}+0 \\
6 \\
\end{array}$ & -0.1 & -0.5 \\
\hline 63 & $\begin{array}{l}\text { CCGTCGTGTTAT } \\
\text { TAAAGACCCCT }\end{array}$ & 23 & 25 & 0.195 & $\begin{array}{c}7.60 \mathrm{E}+ \\
04\end{array}$ & $\begin{array}{c}1.93 \mathrm{E}+ \\
06\end{array}$ & $\begin{array}{c}3.50 \mathrm{E}+0 \\
6\end{array}$ & -0.5 & 0 \\
\hline 63 & $\begin{array}{l}\text { GAGTCAATCGAG } \\
\text { TTTACGTGGCG }\end{array}$ & 23 & 25 & 0.195 & $\begin{array}{c}4.90 \mathrm{E}+ \\
04 \\
\end{array}$ & $\begin{array}{c}1.93 \mathrm{E}+ \\
06 \\
\end{array}$ & $\begin{array}{c}1.43 \mathrm{E}+0 \\
6\end{array}$ & -0.7 & -1.5 \\
\hline 63 & $\begin{array}{l}\text { TTCGGTTCTCTC } \\
\text { CAAAAAAAGCA }\end{array}$ & 23 & 25 & 0.195 & $\begin{array}{c}2.17 \mathrm{E}+ \\
05\end{array}$ & $\begin{array}{c}1.93 \mathrm{E}+ \\
06\end{array}$ & $\begin{array}{c}6.14 \mathrm{E}+0 \\
6\end{array}$ & -0.5 & -0.2 \\
\hline 63 & $\begin{array}{l}\text { TGGCACTTATAG } \\
\text { CTGTCGGAAGA }\end{array}$ & 23 & 25 & 0.195 & $\begin{array}{c}1.58 \mathrm{E}+ \\
05\end{array}$ & $\begin{array}{c}1.93 \mathrm{E}+ \\
06\end{array}$ & $\begin{array}{c}3.23 \mathrm{E}+0 \\
6\end{array}$ & -0.9 & -0.2 \\
\hline 63 & $\begin{array}{l}\text { GAGTCCGCAAA } \\
\text { AATATAGGAGG } \\
\text { C }\end{array}$ & 23 & 25 & 0.195 & $\begin{array}{c}2.20 \mathrm{E}+ \\
06\end{array}$ & $\begin{array}{c}1.93 \mathrm{E}+ \\
06\end{array}$ & $\begin{array}{c}5.32 \mathrm{E}+0 \\
6\end{array}$ & -0.3 & 0.1 \\
\hline 63 & $\begin{array}{c}\text { CGAGAGTCTGTA } \\
\text { ATAGCCGATGC }\end{array}$ & 23 & 25 & 0.195 & $\begin{array}{c}1.06 \mathrm{E}+ \\
06\end{array}$ & $\begin{array}{c}1.93 \mathrm{E}+ \\
06\end{array}$ & $\begin{array}{c}4.68 \mathrm{E}+0 \\
6 \\
\end{array}$ & -0.4 & -0.6 \\
\hline 63 & $\begin{array}{l}\text { GCCTCACATAAC } \\
\text { TGGAGAAACCT }\end{array}$ & 23 & 25 & 0.195 & $\begin{array}{c}1.08 \mathrm{E}+ \\
06\end{array}$ & $\begin{array}{c}1.93 \mathrm{E}+ \\
06\end{array}$ & $\begin{array}{c}3.30 \mathrm{E}+0 \\
6\end{array}$ & -1.2 & -1.4 \\
\hline 63 & GGCTGTCAATTT & 23 & 25 & 0.195 & $2.83 \mathrm{E}+$ & $1.93 \mathrm{E}+$ & $3.61 \mathrm{E}+0$ & -1.1 & -0.7 \\
\hline
\end{tabular}




\begin{tabular}{|c|c|c|c|c|c|c|c|c|c|}
\hline & ATCAGGGAGGC & & & & 06 & 06 & 6 & & \\
\hline 63 & $\begin{array}{l}\text { TTCGCTGATTGT } \\
\text { AGTGTTGCACA }\end{array}$ & 23 & 25 & 0.195 & $\begin{array}{c}7.73 \mathrm{E}+ \\
04\end{array}$ & $\begin{array}{c}1.93 \mathrm{E}+ \\
06\end{array}$ & $\begin{array}{c}3.18 \mathrm{E}+0 \\
6\end{array}$ & -1.2 & -1 \\
\hline 63 & $\begin{array}{l}\text { ATGGGAACCTAA } \\
\text { AAGTGTGGCTA }\end{array}$ & 23 & 25 & 0.195 & $\begin{array}{c}1.96 \mathrm{E}+ \\
05\end{array}$ & $\begin{array}{c}1.93 \mathrm{E}+ \\
06\end{array}$ & $\begin{array}{c}3.58 \mathrm{E}+0 \\
6\end{array}$ & -1.8 & -0.2 \\
\hline 63 & $\begin{array}{c}\text { GCATTGAGGTAT } \\
\text { TGTTGCTCCCA }\end{array}$ & 23 & 25 & 0.195 & $\begin{array}{c}3.00 \mathrm{E}+ \\
06 \\
\end{array}$ & $\begin{array}{c}1.93 \mathrm{E}+ \\
06 \\
\end{array}$ & $\begin{array}{c}1.74 \mathrm{E}+0 \\
6 \\
\end{array}$ & -0.8 & -1 \\
\hline 63 & $\begin{array}{l}\text { CCATCAGGAATG } \\
\text { ACACACACAAA }\end{array}$ & 23 & 25 & 0.195 & $\begin{array}{c}1.47 \mathrm{E}+ \\
06\end{array}$ & $\begin{array}{c}1.93 \mathrm{E}+ \\
06\end{array}$ & $\begin{array}{c}3.85 \mathrm{E}+0 \\
6\end{array}$ & -1.7 & -1.1 \\
\hline 63 & $\begin{array}{l}\text { ATGCACCGGTAA } \\
\text { TATTCCTCTGC }\end{array}$ & 23 & 25 & 0.195 & $\begin{array}{c}9.60 \mathrm{E}+ \\
04\end{array}$ & $\begin{array}{c}1.93 \mathrm{E}+ \\
06\end{array}$ & $\begin{array}{l}2.31 \mathrm{E}+0 \\
5\end{array}$ & -1.1 & -1.9 \\
\hline 63 & $\begin{array}{c}\text { CGCAGGAATTAA } \\
\text { CATGATGAGCG }\end{array}$ & 23 & 25 & 0.195 & $\begin{array}{c}1.07 \mathrm{E}+ \\
05\end{array}$ & $\begin{array}{c}1.93 \mathrm{E}+ \\
06\end{array}$ & $\begin{array}{c}2.41 \mathrm{E}+0 \\
6\end{array}$ & -1 & -1 \\
\hline 63 & $\begin{array}{c}\text { GAAACACTGGAT } \\
\text { ACCTGTGGGAC }\end{array}$ & 23 & 25 & 0.195 & $\begin{array}{c}3.40 \mathrm{E}+ \\
04\end{array}$ & $\begin{array}{c}1.93 \mathrm{E}+ \\
06\end{array}$ & $\begin{array}{c}2.04 \mathrm{E}+0 \\
6\end{array}$ & -3.4 & -1.5 \\
\hline 63 & $\begin{array}{l}\text { GGGATAGAACTC } \\
\text { ACGTACTCCCC }\end{array}$ & 23 & 25 & 0.195 & $\begin{array}{c}1.46 \mathrm{E}+ \\
04\end{array}$ & $\begin{array}{c}1.93 \mathrm{E}+ \\
06\end{array}$ & $\begin{array}{c}1.20 \mathrm{E}+0 \\
5\end{array}$ & -1.5 & -2 \\
\hline 63 & $\begin{array}{c}\text { GGGATCAGTTGT } \\
\text { ACACTCCCTAG }\end{array}$ & 23 & 25 & 0.195 & $\begin{array}{c}5.85 \mathrm{E}+ \\
04\end{array}$ & $\begin{array}{c}1.93 \mathrm{E}+ \\
06\end{array}$ & $\begin{array}{c}1.36 \mathrm{E}+0 \\
6\end{array}$ & -1.9 & -2.2 \\
\hline 63 & $\begin{array}{c}\text { ATGCGTAACACT } \\
\text { CCGTATTGCAT }\end{array}$ & 23 & 25 & 0.195 & $\begin{array}{c}1.03 \mathrm{E}+ \\
04\end{array}$ & $\begin{array}{l}1.93 \mathrm{E}+ \\
06\end{array}$ & $\begin{array}{l}1.96 \mathrm{E}+0 \\
5\end{array}$ & -1.7 & -2 \\
\hline 63 & $\begin{array}{l}\text { GGTCGAAACGTT } \\
\text { ATATTAACGCG }\end{array}$ & 23 & 25 & 0.195 & $\begin{array}{c}1.93 \mathrm{E}+ \\
05\end{array}$ & $\begin{array}{c}1.93 \mathrm{E}+ \\
06\end{array}$ & $\begin{array}{l}2.62 \mathrm{E}+0 \\
6\end{array}$ & -3.7 & -3.8 \\
\hline 64 & TTGGTGATCC & 10 & 20 & 1.0 & $\begin{array}{c}5.00 \mathrm{E}+ \\
06\end{array}$ & $\begin{array}{c}4.12 \mathrm{E}+ \\
06\end{array}$ & $\begin{array}{c}7.63 \mathrm{E}+0 \\
5\end{array}$ & -0.9 & -1.2 \\
\hline 64 & $\begin{array}{l}\text { AGATTAGCAGGT } \\
\text { TTCCCACC }\end{array}$ & 20 & 20 & 1.0 & $\begin{array}{c}3.10 \mathrm{E}+ \\
06\end{array}$ & $\begin{array}{c}1.16 \mathrm{E}+ \\
07\end{array}$ & $\begin{array}{l}2.03 E+0 \\
6\end{array}$ & -2 & -2.8 \\
\hline 64 & TTGGTGATCC & 10 & 50 & 1.0 & $\begin{array}{c}2.50 \mathrm{E}+ \\
07\end{array}$ & $\begin{array}{c}8.35 \mathrm{E}+ \\
06 \\
\end{array}$ & $\begin{array}{c}5.76 \mathrm{E}+0 \\
6\end{array}$ & 1 & 0.7 \\
\hline 64 & $\begin{array}{c}\text { AGATTAGCAGGT } \\
\text { TTCCCACC } \\
\end{array}$ & 20 & 50 & 1.0 & $\begin{array}{c}4.00 \mathrm{E}+ \\
07\end{array}$ & $\begin{array}{c}2.36 \mathrm{E}+ \\
07\end{array}$ & $\begin{array}{c}4.00 \mathrm{E}+0 \\
6 \\
\end{array}$ & 0 & -0.7 \\
\hline 64 & TTGGTGATCC & 10 & 5 & 1.0 & $\begin{array}{c}3.20 \mathrm{E}+ \\
06\end{array}$ & $\begin{array}{c}2.60 \mathrm{E}+ \\
06\end{array}$ & $\begin{array}{c}6.32 \mathrm{E}+0 \\
5\end{array}$ & -1.8 & -2.2 \\
\hline 64 & $\begin{array}{c}\text { AGATTAGCAGGT } \\
\text { TTCCCACC }\end{array}$ & 20 & 5 & 1.0 & $\begin{array}{c}7.90 \mathrm{E}+ \\
05 \\
\end{array}$ & $\begin{array}{c}7.37 \mathrm{E}+ \\
06 \\
\end{array}$ & $\begin{array}{c}2.53 \mathrm{E}+0 \\
6 \\
\end{array}$ & -3 & -3.8 \\
\hline 65 & TATATCCCAT & 10 & 22.6 & 0.25 & $\begin{array}{c}1.41 \mathrm{E}+ \\
06\end{array}$ & $\begin{array}{c}1.11 \mathrm{E}+ \\
06\end{array}$ & $\begin{array}{c}1.23 \mathrm{E}+0 \\
6\end{array}$ & -0.7 & 1.3 \\
\hline 66 & CACGGCTC & 8 & 25.3 & 1.01 & $\begin{array}{c}8.80 \mathrm{E}+ \\
06\end{array}$ & $\begin{array}{c}3.41 \mathrm{E}+ \\
06\end{array}$ & $\begin{array}{c}6.40 \mathrm{E}+0 \\
6\end{array}$ & $\mathrm{NSP}^{3}$ & $\mathrm{NSP}^{3}$ \\
\hline 66 & CACGGCTC & 8 & 6.6 & 1.01 & $\begin{array}{c}4.40 \mathrm{E}+ \\
06\end{array}$ & $\begin{array}{c}1.98 \mathrm{E}+ \\
06\end{array}$ & $\begin{array}{c}7.17 \mathrm{E}+0 \\
6\end{array}$ & $\mathrm{NSP}^{3}$ & $\mathrm{NSP}^{3}$ \\
\hline 66 & CACGGCTC & 8 & 11 & 1.01 & $\begin{array}{c}5.40 \mathrm{E}+ \\
06\end{array}$ & $\begin{array}{c}2.27 \mathrm{E}+ \\
06\end{array}$ & $\begin{array}{c}9.65 \mathrm{E}+0 \\
6\end{array}$ & $\mathrm{NSP}^{3}$ & $\mathrm{NSP}^{3}$ \\
\hline 66 & CACGGCTC & 8 & 15.9 & 1.01 & $\begin{array}{c}6.50 \mathrm{E}+ \\
06\end{array}$ & $\begin{array}{c}2.63 \mathrm{E}+ \\
06\end{array}$ & $\begin{array}{c}8.70 \mathrm{E}+0 \\
6\end{array}$ & $\mathrm{NSP}^{3}$ & $\mathrm{NSP}^{3}$ \\
\hline 66 & CACGGCTC & 8 & 20.1 & 1.01 & $\begin{array}{c}7.00 \mathrm{E}+ \\
06\end{array}$ & $\begin{array}{c}2.97 \mathrm{E}+ \\
06\end{array}$ & $\begin{array}{c}6.82 \mathrm{E}+0 \\
6\end{array}$ & $\mathrm{NSP}^{3}$ & $\mathrm{NSP}^{3}$ \\
\hline 66 & CACAGCAC & 8 & 25.7 & 1.01 & $\begin{array}{c}1.10 \mathrm{E}+ \\
07\end{array}$ & $\begin{array}{c}3.45 \mathrm{E}+ \\
06\end{array}$ & $\begin{array}{c}6.30 \mathrm{E}+0 \\
6 \\
\end{array}$ & $\mathrm{NSP}^{3}$ & 2.6 \\
\hline 66 & CACAGCAC & 8 & 6.9 & 1.01 & $\begin{array}{c}4.30 \mathrm{E}+ \\
06\end{array}$ & $\begin{array}{c}2.00 \mathrm{E}+ \\
06\end{array}$ & $\begin{array}{c}9.58 \mathrm{E}+0 \\
6\end{array}$ & $\mathrm{NSP}^{3}$ & 2.4 \\
\hline
\end{tabular}




\begin{tabular}{|c|c|c|c|c|c|c|c|c|c|}
\hline 66 & CACAGCAC & 8 & 10.8 & 1.01 & $\begin{array}{c}5.30 \mathrm{E}+ \\
06\end{array}$ & $\begin{array}{c}2.26 \mathrm{E}+ \\
06\end{array}$ & $\begin{array}{c}1.00 \mathrm{E}+0 \\
7\end{array}$ & $\mathrm{NSP}^{3}$ & 2.4 \\
\hline 66 & CACAGCAC & 8 & 16.3 & 1.01 & $\begin{array}{c}5.80 \mathrm{E}+ \\
06\end{array}$ & $\begin{array}{c}2.67 \mathrm{E}+ \\
06\end{array}$ & $\begin{array}{c}7.10 \mathrm{E}+0 \\
6\end{array}$ & $\mathrm{NSP}^{3}$ & 2.5 \\
\hline 66 & CACAGCAC & 8 & 20 & 1.01 & $\begin{array}{c}7.30 \mathrm{E}+ \\
06\end{array}$ & $\begin{array}{c}2.96 \mathrm{E}+ \\
06\end{array}$ & $\begin{array}{c}6.34 \mathrm{E}+0 \\
6\end{array}$ & $\mathrm{NSP}^{3}$ & 2.5 \\
\hline
\end{tabular}

${ }^{1}$ Strand 2 was the perfect complement to strand 1.

${ }^{2}$ Calculated using UNAFOLD.

${ }^{3}$ NSP: no structure possible. 
TABLE S2. Kinetic data for surface hybridization, including experimental forward and reverse rate constants, $k_{\mathrm{F}, \mathrm{I}}$ and $k_{\mathrm{R}, \mathrm{I}}$, and corresponding predictions $k_{\mathrm{F}, \mathrm{B}}$ and $k_{\mathrm{R}, \mathrm{B}}$ for the same reaction in solution.

\begin{tabular}{|c|c|c|c|c|c|c|c|c|c|c|}
\hline Ref & probe $^{1}$ & $\begin{array}{c}\text { Length } \\
\text { nt }\end{array}$ & ${ }^{\circ} \mathrm{C}$ & $\begin{array}{c}{\left[\mathrm{Na}^{+}\right]} \\
\mathrm{M}\end{array}$ & $\begin{array}{l}S_{p} \\
\mathrm{~cm}^{-2}\end{array}$ & $\begin{array}{c}k_{\mathrm{F}, \mathrm{I}} \\
\mathrm{M}^{-1} \mathrm{~s}^{-1}\end{array}$ & $\begin{array}{l}k_{R, I} \\
S^{-1}\end{array}$ & $\begin{array}{c}k_{\mathrm{F}, \mathrm{B}}{ }^{2} \\
M^{-1} \mathrm{~s}^{-1}\end{array}$ & $\begin{array}{r}k_{R, B}{ }^{2} \\
S^{-1}\end{array}$ & $\begin{array}{l}\Delta \mathbf{G}^{\circ}{ }_{\mathrm{PP}}^{3} \\
\mathrm{kcal} / \mathrm{mol}\end{array}$ \\
\hline 37 & TGGTTGGG & 8 & 20 & 0.5 & N/A & $1 \mathrm{E}+7^{5}$ & $2 \mathrm{E}-1^{5}$ & $\begin{array}{l}1.9 \mathrm{E}+6 \\
6.0 \mathrm{E}+6\end{array}$ & $\begin{array}{l}1.1 \mathrm{E}-2 \\
3.6 \mathrm{E}-2\end{array}$ & 2.2 \\
\hline 37 & TGGTTGGG & 8 & 20 & 1 & N/A & $2 \mathrm{E}+7^{5}$ & $\begin{array}{c}1.8 \mathrm{E}- \\
1^{5}\end{array}$ & $\begin{array}{l}3.0 \mathrm{E}+6 \\
6.2 \mathrm{E}+6\end{array}$ & $\begin{array}{l}7.5 \mathrm{E}-3 \\
1.6 \mathrm{E}-2 \\
\end{array}$ & 2.0 \\
\hline 36 & ATACATCTA & 9 & 15 & 0.6 & N/A & $2.5 \mathrm{E}+6^{4}$ & $1.2^{4}$ & $\begin{array}{l}2.2 \mathrm{E}+6 \\
4.5 \mathrm{E}+6 \\
\end{array}$ & $\begin{array}{l}4.9 \mathrm{E}-3 \\
9.8 \mathrm{E}-3\end{array}$ & 0.4 \\
\hline 36 & ATACATCTA & 9 & 23 & 0.6 & N/A & $2.1 \mathrm{E}+6^{4}$ & $1.8^{4}$ & $\begin{array}{l}2.8 \mathrm{E}+6 \\
4.6 \mathrm{E}+6 \\
\end{array}$ & $\begin{array}{l}1.4 \mathrm{E}-1 \\
2.4 \mathrm{E}-1 \\
\end{array}$ & 0.5 \\
\hline 65 & TATATCCСАТ & 10 & 23 & 0.25 & N/A & $1.6 \mathrm{E}+6$ & 4.3E-2 & $1.4 \mathrm{E}+6^{7}$ & $4.6 \mathrm{E}-2^{7}$ & -2 \\
\hline 38 & $\begin{array}{l}\text { AGGTCTTCTG } \\
\text { GTCTCCTTTA }\end{array}$ & $20^{6}$ & 22 & 0.92 & 8E12 & $6.4 \mathrm{E}+4$ & $5.0 \mathrm{E}-5$ & $\begin{array}{l}1.2 \mathrm{E}+7 \\
2.1 \mathrm{E}+6\end{array}$ & $\begin{array}{l}1.9 \mathrm{E}-15 \\
3.3 \mathrm{E}-16\end{array}$ & -3.2 \\
\hline 38 & $\begin{array}{l}\text { AGGTCTTCTG } \\
\text { GTCTCCTTTA }\end{array}$ & $20^{6}$ & 45 & 0.92 & 8E12 & $1.9 \mathrm{E}+5$ & $1.2 \mathrm{E}-3$ & $\begin{array}{l}2.1 \mathrm{E}+7 \\
5.8 \mathrm{E}+6 \\
\end{array}$ & $\begin{array}{l}2.8 \mathrm{E}-7 \\
8.0 \mathrm{E}-8 \\
\end{array}$ & -1.4 \\
\hline 38 & $\begin{array}{l}\text { GGTCTTCTGG } \\
\text { TCTCCTTT }\end{array}$ & $18^{6}$ & 22 & 0.92 & 8E12 & $6.0 \mathrm{E}+4$ & $6.8 \mathrm{E}-5$ & $\begin{array}{l}1.0 \mathrm{E}+7 \\
6.0 \mathrm{E}+6\end{array}$ & $\begin{array}{l}2.3 \mathrm{E}-13 \\
1.4 \mathrm{E}-13 \\
\end{array}$ & -3.2 \\
\hline 38 & $\begin{array}{c}\text { GGTCTTCTGG } \\
\text { TCTCСTTT } \\
\end{array}$ & $18^{6}$ & 45 & 0.92 & 8E12 & $2.7 \mathrm{E}+5$ & 3.9E-3 & $\begin{array}{l}1.8 \mathrm{E}+7 \\
7.3 \mathrm{E}+6 \\
\end{array}$ & $\begin{array}{l}6.4 \mathrm{E}-6 \\
2.7 \mathrm{E}-6 \\
\end{array}$ & -0.8 \\
\hline 38 & $\begin{array}{l}\text { GTCTTCTGGT } \\
\text { CTCCTT } \\
\end{array}$ & $16^{6}$ & 22 & 0.92 & 8E12 & $7.0 \mathrm{E}+4$ & $6.1 \mathrm{E}-5$ & $\begin{array}{l}8.5 \mathrm{E}+6 \\
5.3 \mathrm{E}+6 \\
\end{array}$ & $\begin{array}{l}6.9 \mathrm{E}-11 \\
4.3 \mathrm{E}-11 \\
\end{array}$ & -3.2 \\
\hline 38 & $\begin{array}{c}\text { GTCTTCTGGT } \\
\text { CTCСTT }\end{array}$ & $16^{6}$ & 45 & 0.92 & 8E12 & $2.7 \mathrm{E}+5$ & 8.6E-3 & $\begin{array}{l}1.5 \mathrm{E}+7 \\
6.5 \mathrm{E}+6\end{array}$ & $\begin{array}{l}2.7 \mathrm{E}-4 \\
1.2 \mathrm{E}-4\end{array}$ & -0.8 \\
\hline 45 & $\begin{array}{c}\text { TTCCTCGTAG } \\
\text { GCA } \\
\end{array}$ & 13 & 15 & 0.22 & N/A & $6.0 \mathrm{E}+4$ & $3.0 \mathrm{E}-4$ & $\begin{array}{l}9.7 \mathrm{E}+5 \\
3.9 \mathrm{E}+5 \\
\end{array}$ & $\begin{array}{l}3.4 \mathrm{E}-10 \\
1.4 \mathrm{E}-10 \\
\end{array}$ & -5.1 \\
\hline 40 & $\begin{array}{c}\text { TGATAGGGTG } \\
\text { GTGCTTGCGA } \\
\text { GT } \\
\end{array}$ & 22 & 40 & 0.59 & $1.4 \mathrm{E} 11$ & $4.3 \mathrm{E}+5$ & $2.9 \mathrm{E}-4$ & $\begin{array}{l}1.6 \mathrm{E}+7 \\
1.0 \mathrm{E}+7\end{array}$ & $\begin{array}{l}4.4 \mathrm{E}-12 \\
2.8 \mathrm{E}-12\end{array}$ & -3.8 \\
\hline 41 & $\begin{array}{c}\text { TGTGTTTTTG } \\
\text { ATAAACAGTC } \\
\text { GCTT }\end{array}$ & 24 & 50 & 0.7 & 1E11 & $4.0 \mathrm{E}+5$ & $4.0 \mathrm{E}-3$ & $\begin{array}{l}2.5 \mathrm{E}+7 \\
3.7 \mathrm{E}+6\end{array}$ & $\begin{array}{l}5.2 \mathrm{E}-7 \\
7.6 \mathrm{E}-8\end{array}$ & -2 \\
\hline 41 & $\begin{array}{c}\text { TGTGTTTTTG } \\
\text { ATAAACAGTC } \\
\text { GCTT }\end{array}$ & 24 & 50 & 0.7 & $1 \mathrm{E} 12$ & $1.0 \mathrm{E}+5$ & $3.0 \mathrm{E}-4$ & $\begin{array}{l}2.5 \mathrm{E}+7 \\
3.7 \mathrm{E}+6\end{array}$ & $\begin{array}{l}5.2 \mathrm{E}-7 \\
7.6 \mathrm{E}-8\end{array}$ & -2 \\
\hline 41 & $\begin{array}{c}\text { GCTACTCACA } \\
\text { CCGGCATTCT } \\
\text { CACT }\end{array}$ & 24 & 50 & 0.7 & 1E11 & $2.0 \mathrm{E}+5$ & $3.0 \mathrm{E}-4$ & $\begin{array}{l}2.5 \mathrm{E}+7 \\
8.0 \mathrm{E}+6\end{array}$ & $\begin{array}{c}2.0 \mathrm{E}-9 \\
6.3 \mathrm{E}-10\end{array}$ & -4.2 \\
\hline 41 & $\begin{array}{c}\text { GCTACTCACA } \\
\text { CCGGCATTCT } \\
\text { CACT }\end{array}$ & 24 & 50 & 0.7 & 1E12 & $8.0 \mathrm{E}+4$ & $1.0 \mathrm{E}-4$ & $\begin{array}{l}2.5 \mathrm{E}+7 \\
8.0 \mathrm{E}+6\end{array}$ & $\begin{array}{l}2.0 \mathrm{E}-9 \\
6.3 \mathrm{E}-10\end{array}$ & -4.2 \\
\hline 48 & GGGAATTCGT & 10 & 20 & 0.2 & $1.2 \mathrm{E} 13$ & $2.4 \mathrm{E}+4$ & $2.0 \mathrm{E}-2$ & $\begin{array}{l}5.5 \mathrm{E}+5 \\
5.0 \mathrm{E}+6\end{array}$ & $\begin{array}{l}1.7 \mathrm{E}-4 \\
1.5 \mathrm{E}-3\end{array}$ & -5.7 \\
\hline 48 & GGGAATTCGT & 10 & 20 & 0.3 & $1.2 \mathrm{E} 13$ & $4.9 \mathrm{E}+4$ & $5.2 \mathrm{E}-3$ & $\begin{array}{l}1.5 \mathrm{E}+6 \\
5.0 \mathrm{E}+6\end{array}$ & $\begin{array}{l}2.3 \mathrm{E}-4 \\
8.0 \mathrm{E}-4\end{array}$ & -6 \\
\hline 48 & GGGAATTCGT & 10 & 20 & 0.4 & $1.2 \mathrm{E} 13$ & $4.5 \mathrm{E}+4$ & $3.3 \mathrm{E}-3$ & $\begin{array}{l}2.1 \mathrm{E}+6 \\
5.0 \mathrm{E}+6\end{array}$ & $\begin{array}{l}2.1 \mathrm{E}-4 \\
5.1 \mathrm{E}-4\end{array}$ & -6.2 \\
\hline
\end{tabular}




\begin{tabular}{|c|c|c|c|c|c|c|c|c|c|c|}
\hline 48 & GGGAATTCGT & 10 & 20 & 0.5 & $1.2 \mathrm{E} 13$ & $9.6 \mathrm{E}+4$ & $4.0 \mathrm{E}-3$ & $\begin{array}{l}2.6 \mathrm{E}+6 \\
5.0 \mathrm{E}+6\end{array}$ & $\begin{array}{l}1.8 \mathrm{E}-4 \\
3.4 \mathrm{E}-4\end{array}$ & -6.4 \\
\hline 48 & GGGAATTCGT & 10 & 10 & 0.2 & $1.2 \mathrm{E} 13$ & $3.6 \mathrm{E}+4$ & $1.4 \mathrm{E}-3$ & $\begin{array}{l}4.0 \mathrm{E}+5 \\
4.6 \mathrm{E}+6\end{array}$ & $\begin{array}{l}1.2 \mathrm{E}-6 \\
1.4 \mathrm{E}-5\end{array}$ & -7.3 \\
\hline 48 & GGGAATTCGT & 10 & 15 & 0.2 & $1.2 \mathrm{E} 13$ & $5.3 \mathrm{E}+4$ & $1.7 \mathrm{E}-3$ & $\begin{array}{l}4.7 \mathrm{E}+5 \\
4.7 \mathrm{E}+6\end{array}$ & $\begin{array}{l}1.5 \mathrm{E}-5 \\
1.5 \mathrm{E}-4\end{array}$ & -6.5 \\
\hline 48 & GGGAATTCGT & 10 & 25 & 0.2 & $1.2 \mathrm{E} 13$ & $2.9 \mathrm{E}+4$ & $3.4 \mathrm{E}-2$ & $\begin{array}{l}6.2 \mathrm{E}+5 \\
5.4 \mathrm{E}+6\end{array}$ & $\begin{array}{l}1.7 \mathrm{E}-3 \\
1.5 \mathrm{E}-2\end{array}$ & -4.9 \\
\hline 48 & GGGAATTCGT & 10 & 30 & 0.2 & $1.2 \mathrm{E} 13$ & $6.8 \mathrm{E}+4$ & $4.0 \mathrm{E}-2$ & $\begin{array}{l}7.1 \mathrm{E}+5 \\
5.0 \mathrm{E}+6\end{array}$ & $\begin{array}{l}1.7 \mathrm{E}-2 \\
1.2 \mathrm{E}-1\end{array}$ & -4.1 \\
\hline 48 & $\begin{array}{l}\text { TGCCAAGCTT } \\
\text { GGGAATTCGT }\end{array}$ & 20 & 20 & 0.2 & $1.2 \mathrm{E} 13$ & $1.2 \mathrm{E}+5$ & $2.1 \mathrm{E}-4$ & $\begin{array}{l}1.5 \mathrm{E}+6 \\
2.3 \mathrm{E}+6\end{array}$ & $\begin{array}{l}5.0 \mathrm{E}-17 \\
7.5 \mathrm{E}-17\end{array}$ & -15.7 \\
\hline 48 & $\begin{array}{l}\text { GTCCTAGGCC } \\
\text { GCTTAAGCGC } \\
\text { TTTCGAAGCG }\end{array}$ & 30 & 20 & 0.2 & $1.2 \mathrm{E} 13$ & $3.9 \mathrm{E}+5$ & $1.0 \mathrm{E}-5$ & $\begin{array}{l}2.8 \mathrm{E}+6 \\
1.4 \mathrm{E}+6\end{array}$ & $\begin{array}{l}8.8 \mathrm{E}-31 \\
4.3 \mathrm{E}-31\end{array}$ & -19.8 \\
\hline 39 & $\begin{array}{c}\text { CACTTCACTT } \\
\text { TCTTTCCAAG } \\
\text { AG }\end{array}$ & 22 & 21 & 0.12 & $1.4 \mathrm{E} 12$ & $1.1 \mathrm{E}+5$ & N/A & $5.2 \mathrm{E}+5^{7}$ & N/A & -2.3 \\
\hline 39 & $\begin{array}{c}\text { CACTTCACTT } \\
\text { TCTTTCCAAG } \\
\text { AG }\end{array}$ & 22 & 21 & 0.12 & $2.8 \mathrm{E} 12$ & $5.4 \mathrm{E}+4$ & N/A & $5.2 \mathrm{E}+5^{7}$ & N/A & -2.3 \\
\hline 39 & $\begin{array}{c}\text { CACTTCACTT } \\
\text { TCTTTCCAAG } \\
\text { AG }\end{array}$ & 22 & 21 & 0.12 & 5.1E12 & $5.2 \mathrm{E}+4$ & N/A & $5.2 \mathrm{E}+5^{7}$ & N/A & -2.3 \\
\hline 35 & N/A & 30 & 25 & 0.8 & N/A & $4.5 \mathrm{E}+5$ & $7.8 \mathrm{E}-3$ & $2.2 \mathrm{E}+7$ & N/A & N/A \\
\hline 44 & $\begin{array}{l}\text { GTTGTCAAGA } \\
\text { TGCTACCGTT } \\
\text { CAGAG }\end{array}$ & 25 & 20 & 0.5 & 5E12 & $5.7 \mathrm{E}+4$ & N/A & $1.2 \mathrm{E}+6^{7}$ & $5.0 \mathrm{E}-23$ & -3.4 \\
\hline 44 & $\begin{array}{c}\text { GTTGTCAAGA } \\
\text { TGCTACCGTT } \\
\text { CAGAG }\end{array}$ & 25 & 20 & 1 & $5 \mathrm{E} 12$ & $5.5 \mathrm{E}+4$ & N/A & $1.6 \mathrm{E}+6^{7}$ & $3.1 \mathrm{E}-24$ & -3.7 \\
\hline 44 & $\begin{array}{c}\text { AGATCAGTGC } \\
\text { GTCTGTACTA } \\
\text { GCAGT }\end{array}$ & 25 & 20 & 0.5 & 5E12 & $2.4 \mathrm{E}+4$ & N/A & $7.2 \mathrm{E}+5^{7}$ & $1.1 \mathrm{E}-23$ & -6.8 \\
\hline 44 & $\begin{array}{c}\text { AGATCAGTGC } \\
\text { GTCTGTACTA } \\
\text { GCAGT }\end{array}$ & 25 & 20 & 1 & 5E12 & $3.5 \mathrm{E}+4$ & N/A & $8.6 \mathrm{E}+5^{7}$ & $6.3 \mathrm{E}-25$ & -7.7 \\
\hline 44 & $\begin{array}{c}\text { AGATCAGTGC } \\
\text { GTCTGTACTA } \\
\text { GCACA }\end{array}$ & 25 & 20 & 0.5 & 5E12 & $3.2 \mathrm{E}+3$ & N/A & $2.0 \mathrm{E}+5^{7}$ & $1.8 \mathrm{E}-24$ & -12.6 \\
\hline 44 & $\begin{array}{c}\text { AGATCAGTGC } \\
\text { GTCTGTACTA } \\
\text { GCACA }\end{array}$ & 25 & 20 & 1 & 5E12 & $3.5 \mathrm{E}+3$ & N/A & $4.3 \mathrm{E}+5^{7}$ & $1.8 \mathrm{E}-25$ & -13.9 \\
\hline 42 & $\begin{array}{c}\text { TGGATTGCGG } \\
\text { CTGAT }\end{array}$ & 15 & 22 & 0.154 & N/A & $5.4 \mathrm{E}+4$ & N/A & $\begin{array}{c}\mathrm{N} / \mathrm{A}^{8} \\
4.1 \mathrm{E}+6\end{array}$ & $\begin{array}{c}\mathrm{N} / \mathrm{A}^{8} \\
7.4 \mathrm{E}-10\end{array}$ & -5.5 \\
\hline 50 & $\begin{array}{c}\text { CAGGACTGTC } \\
\text { GT }\end{array}$ & 12 & 33 & 0.16 & 6E12 & $1.8 \mathrm{E}+4$ & $2.6 \mathrm{E}-4$ & $\begin{array}{c}\mathrm{N} / \mathrm{A}^{8} \\
4.4 \mathrm{E}+6\end{array}$ & $\begin{array}{c}\text { N/A } \\
1.9 \mathrm{E}-3\end{array}$ & -3.6 \\
\hline 46 & $\begin{array}{c}\text { TGTACATCAC } \\
\text { AACTA }\end{array}$ & 15 & 22 & 0.17 & 8E10 & $2.4 \mathrm{E}+5$ & $1.7 \mathrm{E}-4$ & $\begin{array}{c}\mathrm{N} / \mathrm{A}^{8} \\
1.7 \mathrm{E}+6 \\
\end{array}$ & $\begin{array}{c}\mathrm{N}^{\mathrm{N}} \mathrm{A}^{8} \\
1.1 \mathrm{E}-7 \\
\end{array}$ & -5.0 \\
\hline 46 & $\begin{array}{c}\text { TGTACATCAC } \\
\text { AACTA }\end{array}$ & 15 & 22 & 0.17 & 2.5E11 & $1.2 \mathrm{E}+5$ & $1.1 \mathrm{E}-4$ & $\begin{array}{c}\mathrm{N} / \mathrm{A}^{8} \\
1.7 \mathrm{E}+6\end{array}$ & $\begin{array}{c}\mathrm{N} / \mathrm{A}^{8} \\
1.1 \mathrm{E}-7\end{array}$ & -5.0 \\
\hline
\end{tabular}




\begin{tabular}{|c|c|c|c|c|c|c|c|c|c|c|}
\hline 46 & $\begin{array}{c}\text { TGTACATCAC } \\
\text { AACTA }\end{array}$ & 15 & 22 & 0.17 & $6.7 \mathrm{E} 11$ & $8.4 \mathrm{E}+4$ & $1.0 \mathrm{E}-4$ & $\begin{array}{c}\mathrm{N}^{\mathrm{A}} \mathrm{A}^{8} \\
1.7 \mathrm{E}+6\end{array}$ & $\begin{array}{c}\mathrm{N} / \mathrm{A}^{8} \\
1.1 \mathrm{E}-7\end{array}$ & -5.0 \\
\hline 46 & $\begin{array}{c}\text { TGTACATCAC } \\
\text { AACTA }\end{array}$ & 15 & 22 & 0.17 & $1.1 \mathrm{E} 12$ & $5.6 \mathrm{E}+4$ & $9.0 \mathrm{E}-5$ & $\begin{array}{c}\text { N/A } \\
1.7 \mathrm{E}+6\end{array}$ & $\begin{array}{c}\text { N/A } \\
1.1 \mathrm{E}-7\end{array}$ & -5.0 \\
\hline 47 & $\begin{array}{c}\text { TGTACATCAC } \\
\text { AACTA }\end{array}$ & 15 & 22 & 0.137 & $2.5 \mathrm{E} 12$ & $5.6 \mathrm{E}+4$ & $2.5 \mathrm{E}-5$ & $\begin{array}{c}\text { N/A } \\
1.7 \mathrm{E}+6\end{array}$ & $\begin{array}{c}\text { N/A } \\
1.8 \mathrm{E}-7\end{array}$ & -4.9 \\
\hline 72 & $\begin{array}{c}\text { TGTACATCAC } \\
\text { AACTA }\end{array}$ & 15 & 22 & 0.137 & $2.5 \mathrm{E} 12$ & $1.8 \mathrm{E}+4$ & $1.3 \mathrm{E}-5$ & $\begin{array}{c}\text { N/A } \\
1.7 \mathrm{E}+6\end{array}$ & $\begin{array}{c}\text { N/A } \\
1.8 \mathrm{E}-7\end{array}$ & -4.9 \\
\hline 9 & $\begin{array}{c}\text { CCTACGCCAT } \\
\text { CAGCTCCAAC }\end{array}$ & 20 & 22 & 0.15 & $6.6 \mathrm{E} 11$ & $4.3 \mathrm{E}+4$ & $1.3 \mathrm{E}-4$ & $\begin{array}{c}\text { N/A } \\
2.5 \mathrm{E}+6\end{array}$ & $\begin{array}{c}\text { N/A } \\
1.1 \mathrm{E}-15\end{array}$ & -3.1 \\
\hline
\end{tabular}

${ }^{1}$ Target sequences were fully complementary to the probe sequence.

${ }^{2}$ Unless indicated otherwise (see footnote 7 ), $k_{\mathrm{F}, \mathrm{B}}$ was calculated using (top) the Wetmur-

Davidson and (bottom) the WNV method. Unless indicated otherwise, $k_{\mathrm{R}, \mathrm{B}}$ was then obtained from $k_{\mathrm{F}, \mathrm{B}}$ and the UNAFOLD predicted hybridization equilibrium constant using equation 4 in the main text.

${ }^{3}$ Calculated using UNAFOLD.

${ }^{4}$ Estimated from Fig. 2C in original reference.

${ }^{5}$ Estimated from Fig. 6A of original reference.

${ }^{6}$ For these entries, same complementary 20mer target sequence was used but with one or two nucleotide overhangs, at each end, for the 18mer and 16mer probes, respectively.

${ }^{7}$ Measured experimentally.

${ }^{8} \mathrm{WD}$ estimate is not available as experimental conditions were outside its applicability. 
ADDITIONAL DISCUSSION OF FIGURE 7 (MAIN TEXT). In Figure 7A, the Okahata dataset ${ }^{48}$ indicates an opposite trend to the rest of the studies, with $\Delta E_{\mathrm{A}, \mathrm{F}}$ decreasing as $\Delta G_{\mathrm{PP}}^{0} / N_{\mathrm{BP}}$ becomes more negative. A distinguishing feature of the Okahata dataset is its high probe coverage, with biotinylated probes immobilized to avidin or streptavidin modified supports at 1.2 $\times 10^{13}$ probes $/ \mathrm{cm}^{2}$, corresponding to about a $3 \mathrm{~nm}$ separation between sites and a probe volume fraction of close to $20 \%$. For a completely jam-packed limit, a physical constraint that would have to be obeyed is that steric crowding forces the probes into a parallel orientation. In this li9mit, therefore, probes' ability to arrange into an antiparallel arrangement needed for base pairing would be compromised. These considerations point to conformational constraints as a possible explanation for the contrary trend of the Okahata dataset. Since such an effect should become more severe for longer probes, it could also explain why the two points most responsible for the contrary trend, with $\Delta E_{\mathrm{A}, \mathrm{F}}$ values of 0.74 and 1.72 in Figure 7A, correspond to the two longest sequences at $30 \mathrm{nt}$ and $20 \mathrm{nt}$, respectively. The Okahata dataset also exhibits a distinct behavior in Figure 7B, with a shift to the left suggesting that a threshold in $\Delta G_{\mathrm{PP}}^{0} / N_{\mathrm{BP}}$ needs to be reached before an effect on $\Delta E_{\mathrm{A}, \mathrm{R}}$ is observed. This could similarly be speculated to arise from conformational restrictions where, under dense packing, a threshold in $\Delta G^{\circ}{ }_{\mathrm{pP}} / N_{\mathrm{BP}}$ needs to be reached to overcome costs associated with probes reorienting into an antiparallel arrangement. 
SUPPORTING FIGURES. The below graphs reproduce Figures 5, 6A, and 7 of the main text, but using WD predictions for $k_{\mathrm{F}, \mathrm{B}}$.
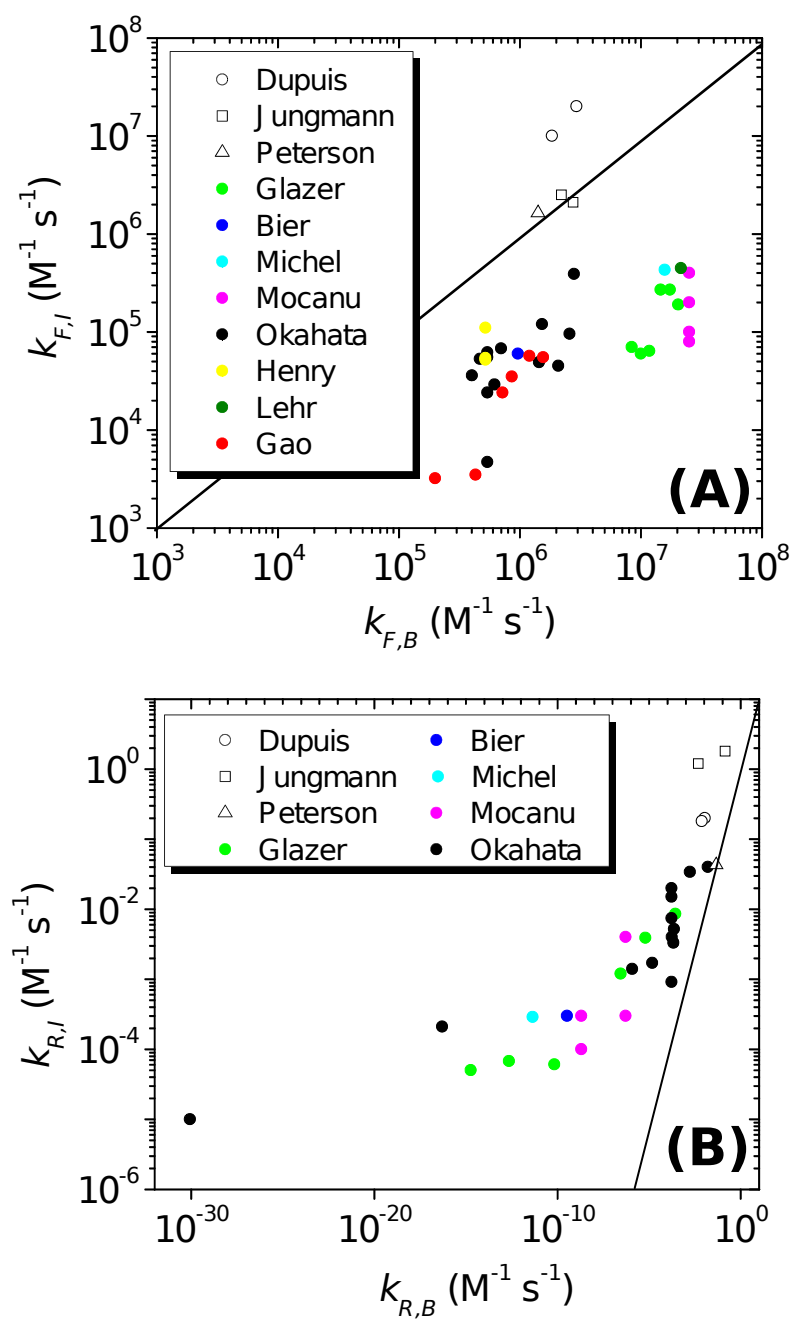

Figure S1. (A). Comparison of forward rate constants for hybridization on solid supports $\left(k_{F, I}\right)$ and in solution $\left(k_{F, B}\right)$. The diagonal line corresponds to $k_{F, I}=k_{F, B}$. Values for $k_{F, B}$ are estimates from the WD model, with the exception of the Peterson, Gao, and Henry datasets for which experimental solution data were available. Experimental $k_{F, I}$ values were compiled from studies 35-41, 44, 45, 48, and 65 whose conditions fell within applicability of the WD model. (B). Comparison of reverse rate constants, $k_{R, I}$ and $k_{R, B}$. The solid line represents condition of equivalence, $k_{R, I}=k_{R, B}$. Values for $k_{R, I}$ are experimental data. Values for $k_{R, B}$ were calculated using equation 4 (main text) with $k_{F, B}$ and $K_{a}$ as inputs, with the exception of the Peterson dataset for which an experimental value was available. 


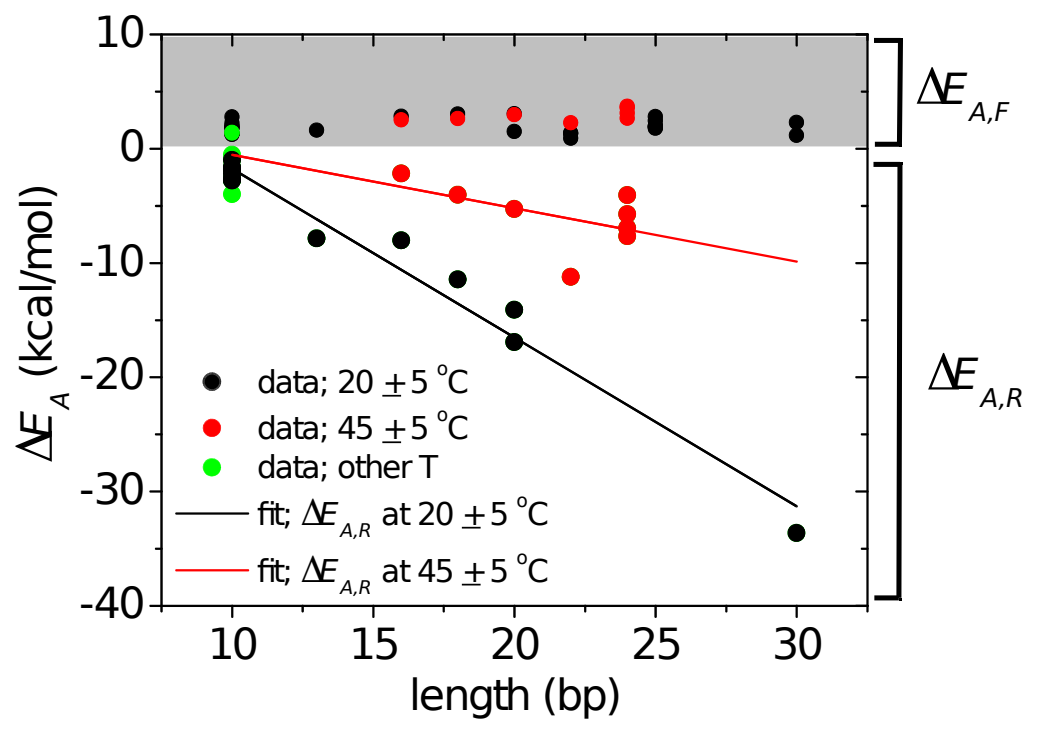

Figure S2. Differences between surface and solution activation energies as a function of duplex length. For the forward direction, $\Delta E_{A, F}$ was estimated from the data of Figure S1A using equation 9 (main text) and is shown in the upper, shaded portion of the figure. $\Delta E_{A, R}$ for the reverse reaction, calculated from Figure S1B data using equation 10 (main text), is shown in the lower unshaded portion. Prediction of solution rate constants used the WD method. The data are further classified by temperature range, either $20 \pm 5{ }^{\circ} \mathrm{C}$ (black series), $45 \pm 5^{\circ} \mathrm{C}$ (red series), or other (green series). Linear fits to $\Delta E_{A, R}$ are shown for both the $20^{\circ} \mathrm{C}$ and $45^{\circ} \mathrm{C}$ series. 
(A)



(B)

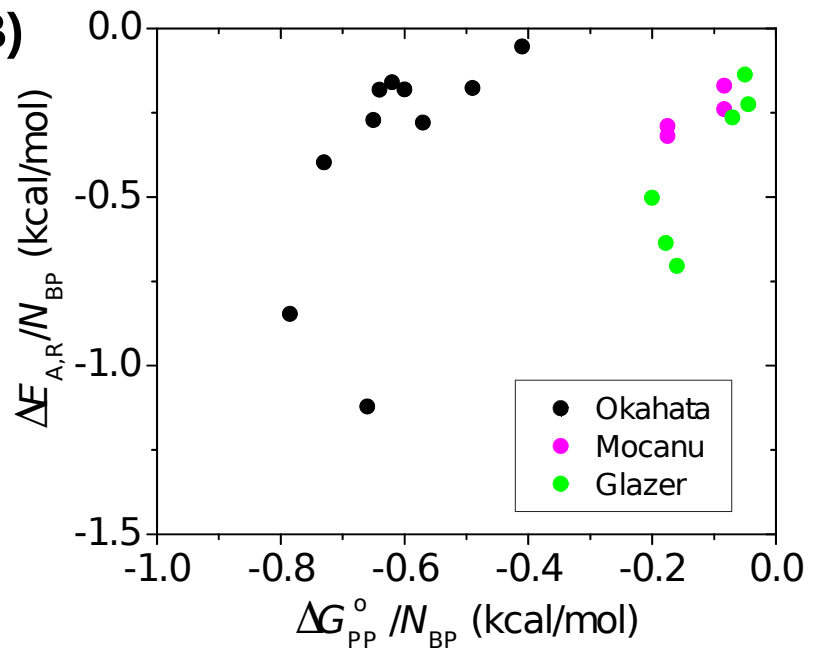

Figure S3. (A). Difference between activation barriers to hybridization on solid supports and in solution, $\Delta E_{\mathrm{A}, \mathrm{F}}$, as a function of competition from probe-probe associations $\Delta G_{\mathrm{PP}}^{0} / N_{\mathrm{BP}}$. All datasets except that of Gao, which provided experimental $k_{F, B}$ values, used WD predictions for $k_{F, B}$ in the calculation of $\Delta E_{\mathrm{A}, \mathrm{F}}$. (B). Offset in the reverse activation barrier per base pair $\Delta E_{\mathrm{A}, \mathrm{R}} /$ $N_{\mathrm{BP}}$, relative to solution, as a function of the probe-probe interaction parameter $\Delta G_{\mathrm{PP}}^{\mathrm{o}} / N_{\mathrm{BP}}$. The Gao dataset was not included as this study did not report $k_{R, I}$ data. 


\section{REFERENCES}

(numbering maintains consistency of Tables S1 and S2 citations with the main text)

(9) Sedighi, A.; Li, P. C. H.; Pekcevik, I. C.; Gates, B. D. A Proposed Mechanism of the Influence of Gold Nanoparticles on DNA Hybridization. ACS Nano 2014, 8 (7), 6765-6777.

(35) Lehr, H.-P.; Reimann, M.; Brandenburg, A.; Sulz, G.; Klapproth, H. Real-Time Detection of Nucleic Acid Interactions by Total Internal Reflection Fluorescence. Anal. Chem. 2003, 75, 2414-2420.

(36) Jungmann, R.; Steinhauer, C.; Scheible, M.; Kuzyk, A.; Tinnefeld, P.; Simmel, F. C. Single-Molecule Kinetics and Super-Resolution Microscopy by Fluorescence Imaging of Transient Binding on DNA Origami. Nano Lett. 2010, 10, 4756-4761.

(37) Dupuis, N. F.; Holmstrom, E. D.; Nesbitt, D. J. Single-Molecule Kinetics Reveal CationPromoted DNA Duplex Formation through Ordering of Single-Stranded Helices. Biophys. J. 2013, 105, 756-766.

(38) Glazer, M.; Fidanza, J. A.; McGall, G. H.; Trulson, M. O.; Forman, J. E.; Suseno, A.; Frank, C. W. Kinetics of Oligonucleotide Hybridization to Photolithographically Patterned DNA Arrays. Anal. Biochem. 2006, 358, 225-238.

(39) Henry, M. R.; Stevens, P. W.; Sun, J.; Kelso, D. M. Real-Time Measurements of DNA Hybridization on Microparticles with Fluorescence Resonance Energy Transfer. Anal. Biochem. 1999, 276, 204-214.

(40) Michel, W.; Mai, T.; Naiser, T.; Ott, A. Optical Study of DNA Surface Hybridization Reveals DNA Surface Density as a Key Parameter for Microarray Hybridization Kinetics. Biophys. J. 2007, 92, 999-1004.

(41) Mocanu, D.; Kolesnychenko, A.; Aarts, S.; Dejong, A. T.; Pierik, A.; Coene, W.; Vossenaar, E.; Stapert, H. Quantitative Analysis of DNA Hybridization in a Flowthrough Microarray for Molecular Testing. Anal. Biochem. 2008, 380, 84-90.

(42) Eze, N. A.; Sullivan, R. S.; Milam, V. T. Analysis of in Situ LNA and DNA Hybridization Events on Microspheres. Biomacromolecules 2017, 18, 1086-1096.

(44) Gao, Y.; Wolf, L. K.; Georgiadis, R. M. Secondary Structure Effects on DNA Hybridization Kinetics: A Solution versus Surface Comparison. Nucl. Acids. Res. 2006, 34, 3370-3377.

(45) Bier, F. F.; Kleinjung, F.; Scheller, F. W. Real-Time Measurement of Nucleic-Acid Hybridization Using Evanescent-Wave Sensors: Steps towards the Genosensor. Sens. Actuator B-Chem. 1997, 38, 78-82.

(46) Xu, F.; Pellino, A. M.; Knoll, W. Electrostatic Repulsion and Steric Hindrance Effects of Surface Probe Density on Deoxyribonucleic Acid (DNA)/Peptide Nucleic Acid (PNA) Hybridization. Thin Solid Films 2008, 516, 8634-8639.

(47) Tawa, K.; Yao, D. F.; Knoll, W. Matching Base-Pair Number Dependence of the Kinetics of DNA-DNA Hybridization Studied by Surface Plasmon Fluorescence Spectroscopy. Biosens. Bioelectron. 2005, 21, 322-329. 
(48) Okahata, Y.; Kawase, M.; Niikura, K.; Ohtake, F.; Furusawa, H.; Ebara, Y. Kinetic Measurements of DNA Hybridisation on an Oligonucleotide-Immobilized 27-MHz Quartz Crystal Microbalance. Anal. Chem. 1998, 70, 1288-1296.

(50) Nava, G.; Ceccarello, E.; Giavazzi, F.; Salina, M.; Damin, F.; Chiari, M.; Buscaglia, M.; Bellini, T.; Zanchetta, G. Label-Free Detection of DNA Single-Base Mismatches Using a Simple Reflectance-Based Optical Technique. Phys. Chem. Chem. Phys. 2016, 18, 13395.

(60) Freier, S. M.; Albergo, D. D.; Turner, D. H. Solvent Effects on the Dynamics of (DGDC) 3 . Biopolymers 1983, 22, 1107-1131.

(61) Nelson, J. W.; Tinoco Jr., I. Comparison of the Kinetics of Ribooligonucleotide, Deoxyribooligonucleeotide, and Hybrid Oligonucleotide Double-Strand Formation by Temperature-Jump Kinetics. Biochemistry 1982, 21, 5289-5295.

(62) Williams, A. P.; Longfellow, C. E.; Freier, S. M.; Kierzek, R.; Turner, D. H. Laser Temperature-Jump, Spectroscopic and Thermodynamic Study of Salt Effects on Duplex Formation by DGCATGC. Biochemistry 1989, 28, 4283-4291.

(63) Hata, H.; Kitajima, T.; Suyama, A. Influence of Thermodynamically Unfavorable Secondary Structures on DNA Hybridization Kinetics. Nucl. Acids Res. 2018, 46 (2), 782-791. (64) Morrison, L. E.; Stols, L. M. Sensitive Fluorescence-Based Thermodynamic and Kinetic Measurements of DNA Hybridization in Solution. Biochemistry 1993, 32, 3095-3104.

(65) Peterson, E. M.; Manhart, M. W.; Harris, J. M. Single-Molecule Fluorescence Imaging of Interfacial DNA Hybridization Kinetics at Selective Capture Surfaces. Analytical Chemistry 2016, 88 (2), 1345-1354.

(66) Rauzan, B.; McMichael, E.; Cave, R.; Sevcik, L. R.; Ostrosky, K.; Whitman, E.; Stegemann, R.; Sinclair, A. L.; Serra, M. J.; Deckert, A. A. Kinetics and Thermodynamics of DNA, RNA, and Hybrid Duplex Formation. Biochemistry 2013, 52, 765-772.

(72) Tawa, K.; Knoll, W. Mismatching Base-Pair Dependence of the Kinetics of DNA-DNA Hybridization Studied by Surface Plasmon Fluorescence Spectroscopy. Nucl. Acids Res. 2004, 32, 2372-2377. 